\title{
Asymptotic Capacity of Orthogonal Multi-Level Amplify-and-Forward Relay Networks
}

\author{
Shu-ping Yeh \\ Department of Electrical Engineering \\ Stanford University \\ Stanford, CA 94305-9510, USA \\ shuping@stanford.edu
}

\author{
Olivier Lévêque \\ Faculté Informatique et Communications \\ Ecole Polytechnique Fédérale de Lausanne \\ 1015 Lausanne, Switzerland \\ olivier.leveque@epfl.ch
}

\author{
John M. Cioffi \\ Department of Electrical Engineering \\ Stanford University \\ Stanford, CA 94305-9510, USA \\ cioffi@stanford.edu
}

\begin{abstract}
This paper analyzes the capacity of a wireless relay network composed of a large number of nodes that operate in an amplify-and-forward mode and that divide into a fixed number of levels. The source and relays are only allowed to operate at given time slots, while the destination can receive signals all the time. The capacity computation relies on the study of products of large random matrices, whose limiting eigenvalue distribution is computed via a set of recursive equations.
\end{abstract}

\section{INTRODUCTION}

This paper investigates a special class of wireless relay networks, the multi-level orthogonal amplified-and-forward relay network. Consider a wireless relay network with onedirectional transmission. There are three groups of nodes: the sources, the destinations and the relays. The sources send messages to destination nodes with assistance from the relay. In practice, there are cases where the sources and destinations are far from each other or there are obstructions between the sources and destinations such that direct transmission from source to destination performs poorly. Relays can be used to improve the capacity in these scenarios. In some cases, multiple levels of relays may be required.

Several configurations of the network are possible. The simplest configuration is the multi-hop network. The relays send a modified version of its received signal from the source to the destination, and the destination decodes the message based on its received signal from the relays. Multi-hop network is illustrated in Figure 1. The other possible configuration is the orthogonal relay network [11]. Sources and each level of relays are allocated orthogonal resources for transmission. The destinations listen all the time, even when the link are very weak. The additional links to the destinations can further improve the system capacity. This paper will focus on the orthogonal relay network configuration. The orthogonal relay network is illustrated in Figure 2.

An operation scheme called amplify-and-forward (AF) is adopted by the relays in this paper. In an AF relay network, the relays simply scale the received signals according to their power constraint and forward the scaled signals to the destinations. The AF operation is a reasonable strategy when relays have a limited computation power and no centralized control nor feedback exists. The AF strategy allows saving of

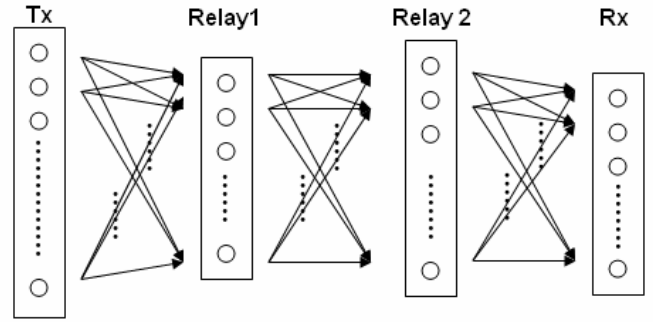

Fig. 1. 2-level multihop AF relay network

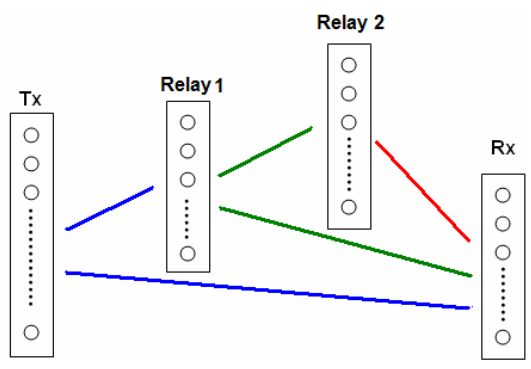

Fig. 2. 2-level orthogonal AF relay network

both computation time and energy at the relays, at the price of noise amplification at each level.

The impact of noise amplification on the system capacity has been quantified for the multihop AF relay network in [5][9], when the number of nodes gets large, but the number of relay levels remains fixed. In [5], general capacity scaling results for the multi-level multihop AF relay network have been derived. This paper adopts the approach in [5] and applies it to obtain a general formula for the multi-level orthogonal $\mathrm{AF}$ relay network. The capacity formula is the expectation of the $\log$ determinant of a product of random matrices. This work needs classical tools from Random Matrix Theory throughout, as developed in [1]-[4].

\section{System Model}

This paper follows the similar system model as in [5]. Flatfading channel, perfectly synchronized transmission and reception among all terminals are assumed. A network with $K$ levels of relays is considered. For completeness, let the source be the $0^{\text {th }}$ level and the destination be the $K+1^{\text {th }}$ level. The 
$k^{t h}$ level consists of $l_{k}$ terminals. Aversion of interference suggests a time-division transmission strategy: during each transmission cycle, there are $K+1$ time slots. At the $k^{t h}$ time slot, $k=1, \ldots, K+1$, the $k-1^{\text {th }}$ level is scheduled to transmit. The message is sent by the sources at the $1^{\text {st }}$ time slot. Then, each level of relay terminals performs the $\mathrm{AF}$ operation; that is, the $k^{t h}$ level of terminals, $k=1, \ldots, K$, will amplify the signal received at $(k-1)^{t h}$ time slot according to its power constraint and send the scaled signal at the $k^{\text {th }}$ time slot. The destination nodes listen all the time and decode the message by combining the signals received during the $K+1$ time slots. $Y_{k}=\left[y_{k 1}, y_{k 2}, \ldots, y_{k l_{k}}\right]^{T}$ and $X_{k}=\left[x_{k 1}, x_{k 2}, \ldots, x_{k l_{k}}\right]^{T}$ denote respectively the received and transmitted signals at the $k^{t h}$ level of relays, $k=1, \ldots, K . X_{0} \in \mathbb{C}^{l_{0} \times 1}$ represent the transmitted signals at the sources, and $Y_{K+1} \in$ $\mathbb{C}^{(K+1) l_{K+1} \times 1}$ are the received signals at the destinations. $Y_{K+1}=\left[Y_{K+1,1}, Y_{K+1,2}, \ldots, Y_{K+1, K+1}\right]^{T}$, where $Y_{K+1, k} \in$ $\mathbb{C}^{l_{K+1} \times 1}$ are the signals received at the destinations, at time slot $k$. The $\mathrm{K}$-level AF orthogonal relay channel can be modeled as:

$$
\begin{aligned}
Y_{k} & =H_{k} X_{k-1}+Z_{k} ; \quad k=1,2, \ldots, K \\
{\left[\begin{array}{c}
Y_{K+1,1} \\
\vdots \\
Y_{K+1, K+1}
\end{array}\right] } & =\left[\begin{array}{c}
\sqrt{\gamma_{1}} H_{K+1,1} X_{0}+Z_{K+1,1} \\
\vdots \\
\sqrt{\gamma_{K+1}} H_{K+1, K+1} X_{K}+Z_{K+1, K+1}
\end{array}\right]
\end{aligned}
$$

where $H_{k}$ denotes the channel matrix between the $(k-1)^{t h}$ and $k^{t h}$ level; $\sqrt{\gamma_{k}} H_{K+1, k}$ denotes the channel matrix between the $(k-1)^{t h}$ level and the destination. The matrices $H_{k}=\left\{H_{k}^{i, j}\right\} \in \mathbb{C}^{l_{k} \times l_{k-1}}$ and $H_{K+1, k}=\left\{H_{K+1, k}^{i, j}\right\} \in$ $\mathbb{C}^{l_{K+1} \times l_{k-1}}$ are independent and their entries are i.i.d. $\mathcal{N}_{\mathbb{C}}(0,1)$ random variables, varying ergodically over time (fast fading assumption). $\gamma_{k}$ accounts for the pathloss from the $k-1^{t h}$ level to the destination. Additive white Gaussian noise $Z_{k}$ (with unit variance) adds at the $k^{t h}$ level. At the destination, $Z_{K+1}=\left[Z_{K+1,1}, \cdots, Z_{K+1, K+1}\right]^{T}$, where $Z_{K+1, k}$ is the destination receiver noise at time slot $k$.

No cooperation nor channel-state information (CSI) is available at the source and relay terminals, but we assume full cooperation and full CSI (i.e. the knowledge of the realizations of all the random matrices $\left.\left\{H_{k}\right\}_{k=1}^{K},\left\{H_{K+1, k}\right\}_{k=1}^{K+1}\right)$ at the destinations.

The power constraint at each node in the network is inversely proportional to the number of nodes at its level. For each level of relays, the received signals are scaled according to the power constraint at this level of relays. The scaling factor of the $k^{t h}$ level of relay is $\alpha_{k}$. Therefore, the scaling is

$$
X_{k}=\alpha_{k} Y_{k}, \quad k=1, \ldots, K
$$

$P_{k}$ is the total power constraint on the $k^{t h}$ level, then

$$
P_{k}=\mathbb{E}\left[X_{k}^{H} X_{k}\right]=\alpha_{k}^{2} \mathbb{E}\left[\left(H_{k} X_{k-1}+Z_{k}\right)^{H}\left(H_{k} X_{k-1}+Z_{k}\right)\right]
$$

Since $X_{k-1}, H_{k}$ and $Z_{k}$ are independent,

$$
\begin{aligned}
P_{k} & =\alpha_{k}^{2}\left(\mathbb{E}\left[X_{k-1}^{H} \mathbb{E}\left[H_{k}^{H} H_{k}\right] X_{k-1}\right]+\mathbb{E}\left[Z_{k}^{H} Z_{k}\right]\right) \\
& =\alpha_{k}^{2}\left(\mathbb{E}\left[X_{k-1}^{H}\left(l_{k} I\right) X_{k-1}\right]+l_{k}\right)=\alpha_{k}^{2} l_{k}\left(P_{k-1}+1\right)
\end{aligned}
$$

$\beta_{k}=\frac{P_{k-1}+1}{P_{k}}$ is the power ratio, $k=1, \ldots, K$. The scaling factors can then be written as $\alpha_{k}=\sqrt{1 / \beta_{k} l_{k}}, k=0, \ldots, K$. For completeness, define $\beta_{0}=\sqrt{1 / P_{0}}, \alpha_{0}=\sqrt{P_{0} / l_{0}}$ and $Y_{0}=\frac{1}{\alpha_{0}} X_{0}$.

To simplify notations, we introduce $\left\{G_{k}\right\}_{k=1}^{K+1}$ and $\left\{Z_{k}^{\prime}\right\}_{k=1}^{K+1}$ for the equivalent representation of the channel between $Y_{k-1}$ and $\left[Y_{K+1, k}, \ldots, Y_{K+1, K+1}\right]^{T}$.

$$
\left[\begin{array}{c}
Y_{K+1, k} \\
\vdots \\
Y_{K+1, K+1}
\end{array}\right]=G_{k} Y_{k-1}+Z_{k}^{\prime}, \quad k=1, \ldots, K+1
$$

It can be shown that $\left\{G_{k}\right\}_{k=1}^{K+1}$ and $\left\{Z_{k}^{\prime}\right\}_{k=1}^{K+1}$ satisfy

$$
G_{K+1}=\alpha_{K} \sqrt{\gamma_{K+1}} H_{K+1, K+1} ; \quad Z_{K+1}^{\prime}=Z_{K+1, K+1} .
$$

For $k=K, \ldots, 2,1$,

$$
G_{k}=\alpha_{k-1}\left[\begin{array}{c}
\sqrt{\gamma_{k}} H_{K+1, k} \\
G_{k+1} H_{k}
\end{array}\right] ; Z_{k}^{\prime}=\left[\begin{array}{c}
Z_{K+1, k} \\
G_{k+1} Z_{k}+Z_{k+1}^{\prime}
\end{array}\right]
$$

The overall channel is then

$$
Y_{K+1}=G_{1} Y_{0}+Z_{1}^{\prime}=\frac{1}{\alpha_{0}} G_{1} X_{0}+Z_{1}^{\prime}
$$

We call $G_{1} Y_{0}$ the signal part and the $Z_{1}^{\prime}$ the noise part.

Let $\Sigma_{k}$ be the expected covariance of $Z_{k}^{\prime}$ given that the destinations have perfect knowledge of the channels. The matrices $\left\{\Sigma_{k}\right\}_{k=1}^{K+1}$ have the following recursive relationship:

$\Sigma_{K+1}=I ; \Sigma_{k-1}=\left[\begin{array}{cc}I & 0 \\ 0 & \Sigma_{k}+G_{k} G_{k}^{H}\end{array}\right], k=K+1 \ldots, 2$.

$\Sigma_{1}$ is the covariance matrix of the noise part.

For the Gaussian noise channel, the capacity is achieved when the entries of $X_{0}$ are jointly Gaussian. Since there is no CSI at the sources and the entries of $H_{1}, H_{K+1,1}$ are i.i.d. Gaussian and independent of $G_{2},[10]$ relates that the optimal $X_{0}$ is distributed according to $\mathcal{N}_{\mathbb{C}}\left(0, \frac{P_{0}}{l_{l}} I\right)$. Thus, the covariance matrix of the signal part is $G_{1} G_{1}^{H}$.

The overall capacity can then be computed as:

$$
\begin{aligned}
C & =\frac{1}{K+1} I\left(X_{0} ; Y_{K+1} \mid G_{1}\right) \\
& =\frac{1}{K+1} \mathbb{E} \log \operatorname{det}\left(I+\Sigma_{1}^{-\frac{1}{2}} G_{1} G_{1}^{H} \Sigma_{1}^{-\frac{1}{2}}\right) \\
& =\frac{1}{K+1} \mathbb{E} \log \operatorname{det}\left(I+G_{1}^{H} \Sigma_{1}^{-1} G_{1}\right)
\end{aligned}
$$

(The $\frac{1}{K+1}$ term comes from the time-division scheme.) To analyze the capacity scaling behavior of the multi-level amplifiedand-forward relay channel, we take all $l_{k}$ 's to infinity. We 
assume they tend to some given ratios while going to infinity, say, $\frac{l_{k}}{l_{K+1}} \rightarrow c_{k}, k=0, \ldots, K$.

\section{CAPACITY ANALYSIS}

Stieltjes transform is a powerful tool for analyzing the limiting eigenvalue distribution (LED) of large dimensional random matrices. A thorough discussion of its applications can be found in [2]. With $F$ as a distribution on $\mathbb{R}$ (here and in the rest of the paper, one identifies a distribution on $\mathbb{R}$ with its cumulative distribution function), the Stieltjes transform is defined as

$$
g(z) \equiv \int_{-\infty}^{\infty} \frac{1}{x-z} d F(x), z \in \mathbb{C}^{+} \equiv\{z \in \mathbb{C}: \operatorname{Im}(z)>0\}
$$

with the inversion formula

$$
\lim _{\epsilon \downarrow 0} \int_{x_{1}}^{x_{2}} \frac{1}{\pi} \operatorname{Im}(g(x+i \epsilon)) d x=F\left(x_{2}\right)-F\left(x_{1}\right) .
$$

A sequence of distributions converges to a limit if and only if the corresponding sequence of Stieltjes transforms converges. The following result is a straightforward consequence of a result by Silverstein [4]. The empirical eigenvalue distribution of an $n \times n$ Hermitian matrix $A_{n}$ with real eigenvalues $\lambda_{1}, \ldots, \lambda_{n}$ is given by $F_{A_{n}}(x)=\frac{1}{n} \sum_{k=1}^{n} 1_{\left\{x \geq \lambda_{k}\right\}}$.

Theorem 1: Let $n, N \geq 1$ and let us assume that:

(a) $X_{n}=\left(X_{i j}^{n}\right)$ is an $n \times N$ random matrix with i.i.d. entries such that $\mathbb{E}\left|X_{11}^{n}-\mathbb{E} X_{11}^{n}\right|^{2}=1$;

(b) $N=N(n)$ with $n / N \rightarrow c>0$ as $n \rightarrow \infty$;

(c) $T_{n}$ is an $n \times n$ random Hermitian non-negative definite matrix such that its empirical eigenvalue distribution $F_{T_{n}}$ converges almost surely, as $n \rightarrow \infty$, to a (deterministic) distribution $F_{T}$, with corresponding Stieltjes transform $g_{T}$;

(d) $X_{n}$ and $T_{n}$ are independent.

Let $A_{n}=\frac{1}{N} X_{n}^{*} T_{n} X_{n}$. Then its empirical eigenvalue distribution $F_{A_{n}}$ converges almost surely, as $n \rightarrow \infty$, to a (deterministic) distribution $F_{A}$, whose Stieltjes transform $g_{A}$ satisfies

$$
z g_{A}(z)+1=c\left(\frac{-1}{g_{A}(z)} g_{T}\left(\frac{-1}{g_{A}(z)}\right)+1\right)
$$

in the sense that, for each $z \in \mathbb{C}^{+}, g=g_{A}(z)$ is the unique solution to (7) such that $g \in \mathbb{C}^{+}$.

In the particular case where $T_{n}=I$, the above equation becomes

$$
z g_{A}(z)+1=\frac{c g_{A}(Z)}{1+g_{A}(z)}
$$

and its solution is the Stieltjes transform of the well-known Marčenko-Pastur distribution [1].

To illustrate how Silverstein's theorem is used to solve the capacity expression (4), the single-level AF relay network is examined first. The single-level case provides general guidance for computing the general multi-level problem. A set of recursive equations are then derived to compute the Stieltjes transform of the LED of the multi-level AF relay network.

\section{A. Single-Level Orthogonal AF Relay Network}

When $K=1, G_{2}=\alpha_{1} \sqrt{\gamma_{2}} H_{2,2}, \Sigma_{2}=I$.

$$
G_{1}=\alpha_{0}\left[\begin{array}{c}
\sqrt{\gamma_{1}} H_{2,1} \\
G_{2} H_{1}
\end{array}\right], \quad \Sigma_{1}=\left[\begin{array}{cc}
I & O \\
O & \Sigma_{2}+G_{2} G_{2}^{H}
\end{array}\right]
$$

From (4), $C_{1 \text {-level orth AF }}=\frac{1}{2} \mathbb{E} \log \operatorname{det}\left(I+G_{1}^{H} \Sigma_{1}^{-1} G_{1}\right)$, where

$$
\begin{aligned}
& G_{1}^{H} \Sigma_{1}^{-1} G_{1}=\frac{1}{l_{0}}\left[\begin{array}{ll}
H_{2,1}^{H} & H_{1}^{H}
\end{array}\right] T_{1}\left[\begin{array}{c}
H_{2,1} \\
H_{1}
\end{array}\right] \\
& T_{1}=\frac{1}{\beta_{0}}\left[\begin{array}{cc}
\gamma_{1} I & O \\
O & G_{2}^{H}\left(\Sigma_{2}+G_{2} G_{2}^{H}\right)^{-1} G_{2}
\end{array}\right] \\
& G_{2}^{H} \Sigma_{2}^{-1} G_{2}=\frac{\gamma_{2}}{\beta_{1} l_{1}} H_{2,2}^{H} H_{2,2}
\end{aligned}
$$

Theorem 2: For matrices $G_{1}^{H} \Sigma_{1}^{-1} G_{1}, G_{2}^{H} \Sigma_{2}^{-1} G_{2}$ and $T_{1}$ satisfying (9), (10) and (11) with $\frac{l_{0}}{l_{2}} \rightarrow c_{0}$ and $\frac{l_{1}}{l_{2}} \rightarrow c_{1}$, the Stieltjes transform $g_{k}$ of the LED of $G_{k}^{H} \Sigma_{k}^{-1} G_{k}$ satisfies:

$$
\begin{aligned}
c_{0}\left(z g_{1}(z)+1\right) & =\frac{\gamma_{1} g_{1}(z)}{\beta_{0}+\gamma_{1} g_{1}(z)}+ \\
\frac{c_{1} g_{1}(z)}{\beta_{0}+g_{1}(z)} & \left(\frac{-\beta_{0}}{\beta_{0}+g_{1}(z)} g_{2}\left(\frac{-\beta_{0}}{\beta_{0}+g_{1}(z)}\right)+1\right) \\
c_{1}\left(z g_{2}(z)+1\right) & =\frac{\gamma_{2} g_{2}(z)}{\beta_{1}+\gamma_{2} g_{2}(z)}
\end{aligned}
$$

where $g_{1}: \mathbb{C}^{+} \rightarrow \mathbb{C}^{+}$and $g_{2}: \mathbb{C}^{+} \rightarrow \mathbb{C}^{+}$.

Proof: The basic proof idea goes as follows. From (11), the eigenvalue distribution of $G_{2}^{H} \Sigma_{2}^{-1} G_{2}$ converges a.s. to a scaled version of the Marčenko-Pastur distribution. The corresponding Stieltjes transform $g_{2}$ can be shown to satisfy (13) with $g_{2}: \mathbb{C}^{+} \rightarrow \mathbb{C}^{+}$. For (9), Theorem 1 applies to compute the LED of $G_{1}^{H} \Sigma_{1}^{-1} G_{1}$, if $T_{1}$ is random Hermitian nonnegative definite and independent of $\left[H_{2,1} H_{1}\right]^{T}$, with its eigenvalue distribution converging almost surely as $l_{0} \rightarrow \infty$. It is obvious that $\frac{l_{2}}{l_{1}+l_{2}}$ of the eigenvalues of $T_{1}$ are equal to $\gamma_{1} / \beta_{0}$ and that the distribution of the other eigenvalues of $T_{1}$ depends on $\frac{1}{\beta_{0}} G_{2}^{H}\left(\Sigma_{2}+G_{2} G_{2}^{H}\right)^{-1} G_{2}$. Therefore, the missing link is the relationship between the eigenvalues of $G_{2}^{H}\left(\Sigma_{2}+G_{2} G_{2}^{H}\right)^{-1} G_{2}$ and $G_{2}^{H} \Sigma_{2}^{-1} G_{2}$. This relationship can be obtained by combining Lemmas 1 and 2 in [5]. From [5],

Lemma 1: 1) If $\Sigma$ is positive definite, then

$G^{H}\left(\Sigma+G G^{H}\right)^{-1} G=G^{H} \Sigma^{-\frac{1}{2}}\left(I+\Sigma^{-\frac{1}{2}} G G^{H} \Sigma^{-\frac{1}{2}}\right)^{-1} \Sigma^{-\frac{1}{2}} G$

2) Let $T=G^{H}\left(\Sigma+G G^{H}\right)^{-1} G \in \mathbb{C}^{l \times l}$ and denote by $t_{i}$ and $m_{i}$ the eigenvalues of $T$ and $G^{H} \Sigma^{-1} G$ respectively, where $t_{1} \leq t_{2} \leq \ldots \leq t_{l}$ and $m_{1} \leq m_{2} \leq \ldots \leq m_{l}$. Then

$$
t_{k}=\frac{m_{k}}{1+m_{k}} \quad k=1, \ldots, l
$$

From Lemma 1, the eigenvalue distributions of $T_{1}$ and $G_{2}^{H} \Sigma_{2}^{-1} G_{2}$ satisfy

$$
\left(c_{1}+1\right) F_{T_{1}}\left(\frac{x}{\beta_{0}}\right)=I_{\left\{x \geq \gamma_{1}\right\}}+c_{1} F_{G_{2}^{H} \Sigma_{2}^{-1} G_{2}}\left(\frac{x}{1-x}\right)
$$


and their corresponding Stieltjes transforms satisfy

$$
\begin{aligned}
& \left(c_{1}+1\right)\left(z g_{T_{1}}(z)+1\right) \\
& =\frac{\gamma_{1}}{\gamma_{1}-\beta_{0} z}+\frac{c_{1}}{1-\beta_{0} z}\left(\frac{\beta_{0} z}{1-\beta_{0} z} g_{2}\left(\frac{\beta_{0} z}{1-\beta_{0} z}\right)+1\right)
\end{aligned}
$$

Since $G_{2}^{H} \Sigma_{2}^{-1} G_{2}$ is Hermitian nonnegative definite, $T_{1}$ is also Hermitian nonnegative definite, and its eigenvalue distribution converges a.s. Therefore, Theorem 1 applies to find the Stieltjes transform $g_{1}$ : combining (7) and (16) yields the conclusion that $g_{1}$ satisfies (12) with $g_{1}: \mathbb{C}^{+} \rightarrow \mathbb{C}^{+}$.

$g_{1}$ can be only be solved numerically. The polynomial equation obtained by combining (12) and (13) is of order 6 and only one of the roots of this equation satisfies both $g_{1}: \mathbb{C}^{+} \rightarrow \mathbb{C}^{+}$ and $g_{2}: \mathbb{C}^{+} \rightarrow \mathbb{C}^{+}$. From $g_{1}$, the inverse Stieltjes transform (6) provides the corresponding LED $F_{1}$. The single-level AF channel capacity is then given by

$$
C_{1 \text {-level orth } \mathrm{AF}} \sim \frac{l_{0}}{2} \int \log (1+x) d F_{1}(x)
$$

as $l_{0} \rightarrow \infty$. Therefore, the capacity increases linearly with the number of nodes at each level.

\section{B. Multi-Level Orthogonal AF Relay Network}

The generalization of the previous result to the multi-level case requires a recursive transformation of the matrices as well as an iterative application of Lemma 1 and Theorem 1 to compute the Stieltjes transform of the LED of the matrix in (4). By introducing a new series of matrices $\left\{T_{k}\right\}_{k=1}^{K}$, we can generalize (9), (10) and (11) to the K-level case. $\left\{T_{k}\right\}$ is defined as

$$
T_{k}=\frac{1}{\beta_{k-1}}\left[\begin{array}{cc}
\gamma_{k} I & O \\
O & G_{k+1}^{H}\left(\Sigma_{k+1}+G_{k+1} G_{k+1}^{H}\right)^{-1} G_{k+1}
\end{array}\right]
$$

The series of matrices $G_{k}^{H} \Sigma_{k}^{-1} G_{k}$ can be then expressed in terms of $T_{k}$.

$$
\begin{aligned}
& G_{k}^{H} \Sigma_{k}^{-1} G_{k}=\frac{1}{l_{k-1}}\left[H_{K+1, k}^{H} H_{k}^{H}\right] T_{k}\left[\begin{array}{c}
H_{K+1, k} \\
H_{k}
\end{array}\right] \\
& G_{K+1}^{H} \Sigma_{K+1}^{-1} G_{K+1}=\frac{\gamma_{K+1}}{\beta_{K} l_{K}} H_{K+1, K+1}^{H} H_{K+1, K+1}
\end{aligned}
$$

Again, capacity computation is through the LED of $G_{1}^{H} \Sigma_{1}^{-1} G_{1}$. The similarity between (9), (10) and (18), (19) suggests a recursive way to compute the Stieltjes transform of the LED of $G_{1}^{H} \Sigma_{1}^{-1} G_{1}$ for the multi-level case.

Theorem 3: For matrix series $\left\{G_{k}^{H} \Sigma_{k}^{-1} G_{k}\right\}_{k=1}^{K+1}$ and $\left\{T_{k}\right\}_{k=1}^{K}$ satisfying (18), (19) and (20), the Stieltjes transforms $g_{k}$ of the LED of $G_{k}^{H} \Sigma_{k}^{-1} G_{k}$ satisfy the following equations.

For $k=1, \ldots, K$ :

$$
\begin{aligned}
c_{k-1}\left(z g_{k}(z)+1\right) & =\frac{\gamma_{k} g_{k}(z)}{\beta_{k-1}+\gamma_{k} g_{k}(z)}+\frac{c_{k} g_{k}(z)}{\beta_{k-1}+g_{k}(z)}+ \\
\frac{c_{k} g_{k}(z)}{\beta_{k-1}+g_{k}(z)} \frac{-\beta_{k-1}}{\beta_{k-1}+g_{k}(z)} g_{k+1}\left(\frac{-\beta_{k-1}}{\beta_{k-1}+g_{k}(z)}\right) & \\
c_{K}\left(z g_{K+1}(z)+1\right) & =\frac{\gamma_{K+1} g_{K+1}(z)}{\beta_{K}+\gamma_{K+1} g_{K+1}(z)}
\end{aligned}
$$

where $g_{k}: \mathbb{C}^{+} \rightarrow \mathbb{C}^{+}, k=1, \ldots, K+1$.

Proof: From Theorem 1, the eigenvalue distribution of $G_{k}^{H} \Sigma_{k}^{-1} G_{k}$ converges a.s. if $T_{k}$ is Hermitian nonnegative definite and the eigenvalue distribution of $T_{k}$ converges a.s., $k=1, \ldots, K$. (18) implies that $T_{k}$ is Hermitian nonnegative definite. The relationship between the eigenvalues of $G_{k+1}^{H} \Sigma_{k+1}^{-1} G_{k+1}$ and $T_{k}$ is provided in Lemma 1. As long as the eigenvalue distribution of $G_{k+1}^{H} \Sigma_{k+1}^{-1} G_{k+1}$ converges a.s., then the eigenvalue distribution of $T_{k}$ converges a.s. For $G_{K+1}^{H} \Sigma_{K+1}^{-1} G_{K+1}$, (20) indicates that its eigenvalue distribution converges a.s. to a scaled version of the MarčenkoPastur distribution. It can be shown that the corresponding Stieltjes transform $g_{K+1}$ satisfies (13) with $g_{K+1}$ : $\mathbb{C}^{+} \rightarrow \mathbb{C}^{+}$. The convergence of the eigenvalue distribution of $G_{K+1}^{H} \Sigma_{K+1}^{-1} G_{K+1}$ ensures that the eigenvalue distribution of $T_{K}$ converges a.s. Consequently, the eigenvalue distribution of $G_{K}^{H} \Sigma_{K}^{-1} G_{K}$ converges, and so does $T_{K-1}$, and so on. By induction, we conclude that the eigenvalue distribution of $G_{k}^{H} \Sigma_{k}^{-1} G_{k}, k=1, \ldots, K+1$ converges almost surely.

As a result, combining (7), (8) and (16), the recursive equations (19) and (20) for the Stieltjes transform $g_{k}$ of the LED of $G_{k}^{H} \Sigma_{k}^{-1} G_{k}, k=1, \ldots K+1$ can be derived.

Computing the capacity (4) requires the knowledge of the LED of $G_{k}^{H} \Sigma_{k}^{-1} G_{k}$. Explicitly, through introduction of a new sequence $\left\{z_{k}\right\}_{k=1}^{K+1}$, where $z_{1}=z, z_{k+1}=\frac{-\beta_{k}}{\beta_{k}+g_{k}\left(z_{k}\right)}$, $k=1, \cdots, K$. (19) becomes, for $k=1, \ldots, K$ :

$$
\begin{array}{r}
g_{k+1}\left(z_{k+1}\right)=\frac{\beta_{k-1}+g_{k}(z)}{\beta_{k-1}}\left(1+\frac{\gamma_{k}}{c_{k}} \cdot \frac{\beta_{k-1}+g_{k}\left(z_{k}\right)}{\beta_{k-1}+\gamma_{k} g_{k}\left(z_{k}\right)}\right)- \\
\frac{c_{k-1}\left(\beta_{k-1}+g_{k}\left(z_{k}\right)\right)^{2}\left(z_{k} g_{k}\left(z_{k}\right)+1\right)}{c_{k} \beta_{k-1} g_{k}\left(z_{k}\right)}
\end{array}
$$

Therefore, $g_{k}\left(z_{k}\right), k=2, \ldots, K+1$, can be expressed in terms of $z$ and $g_{1}(z)$. Substitute $g_{K+1}\left(z_{K+1}\right)$ in terms of $z$ and $g_{1}(z)$ into (20) yields an equation for $g_{1}(z)$. The overall equation for $g_{1}$ is of (very) high order when $K \geq 2$. The root has to be solved numerically and should satisfy the constraints, $g_{k}\left(z_{k}\right) \in \mathbb{C}^{+}, \forall k=1, \ldots, K+1$. The LED $F_{1}$ of $G_{1}^{H} \Sigma_{1}^{-1} G_{1}$ is computed via the inverse Stieltjes transform (6). The capacity is given by

$$
C_{\mathrm{K} \text {-level orth } \mathrm{AF}} \sim \frac{l_{0}}{K+1} \int \log (1+x) d F_{1}(x)
$$

as $l_{0} \rightarrow \infty$. The capacity scales linearly with the number of nodes at each level. Finally, it is possible to show that when $c_{k} \rightarrow \infty, k=1, \ldots, K$ and $c_{0}$ remains fixed, $F_{1}$ converges to a special distribution which can viewed as a combination of $K+1$ orthogonal direct-link MIMO channels with different channel gain factors.

\section{Numerical Simulations}

The theoretical LED obtained in Section III-B approximates the empirical eigenvalue distribution of finite-dimension matrices fairly well. Figure 3 shows that there is indeed a good agreement between the two, even for a small number of nodes 


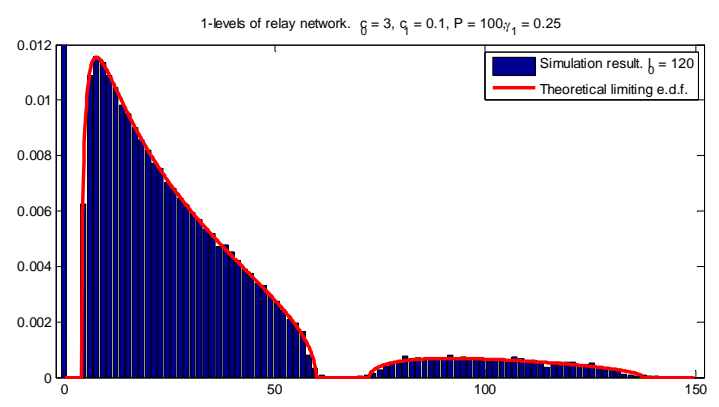

Fig. 3. Limiting versus empirical eigenvalue distribution

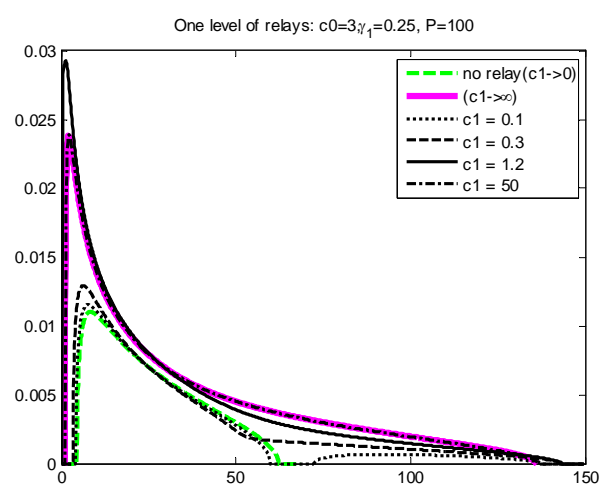

Fig. 4. Varying the number of relays in a 1-level AF relay network: LED

in the network. The value of $\gamma_{1}$ is set to be 0.25 in all of our simulations.

Also of interest is the effect of the number of relays upon the LED and the capacity. The ratio between relay nodes and source nodes varies, while the ratio between the number of source and destination nodes is fixed. When there is only one level of relays, the LED and the capacity are shown in Figures 4 and 5 respectively.

Figure 6 summarizes the comparison between direct-link transmission, multihop relay and orthogonal relay networks. A higher density at large eigenvalues implies a better capacity performance. We can see that as the number of nodes at the relay increases, multihop transmission starts to outperform the direct-link transmission. In all cases, orthogonal relay operation provides the highest capacity.

\section{COnClusion}

The capacity of a large scale multi-level orthogonal AF relay network can be expressed by means of the limiting eigenvalue distribution of a random matrix, whose Stieltjes transform is shown to satisfy a set of recursive equations. A general procedure for solving explicitly these equations has been proposed, from which one can deduce both the limiting eigenvalue distribution and the capacity. From the capacity expression, we conclude that the capacity of the multi-level orthogonal AF relay network scales linearly as the number of nodes goes to infinity, with fixed ratios between all levels.

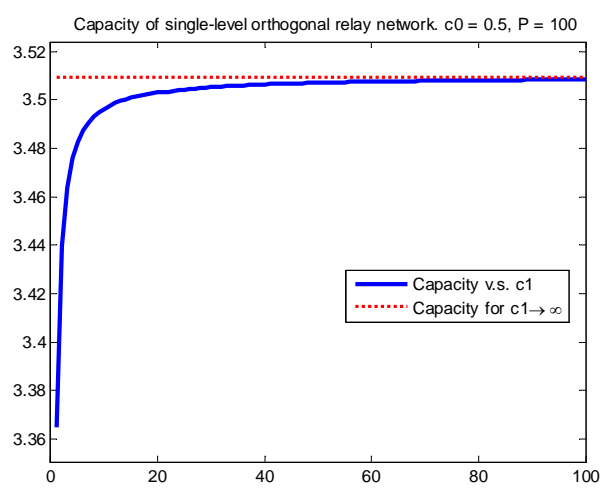

Fig. 5. Varying the number of relays in a 1-level AF relay network: Capacity
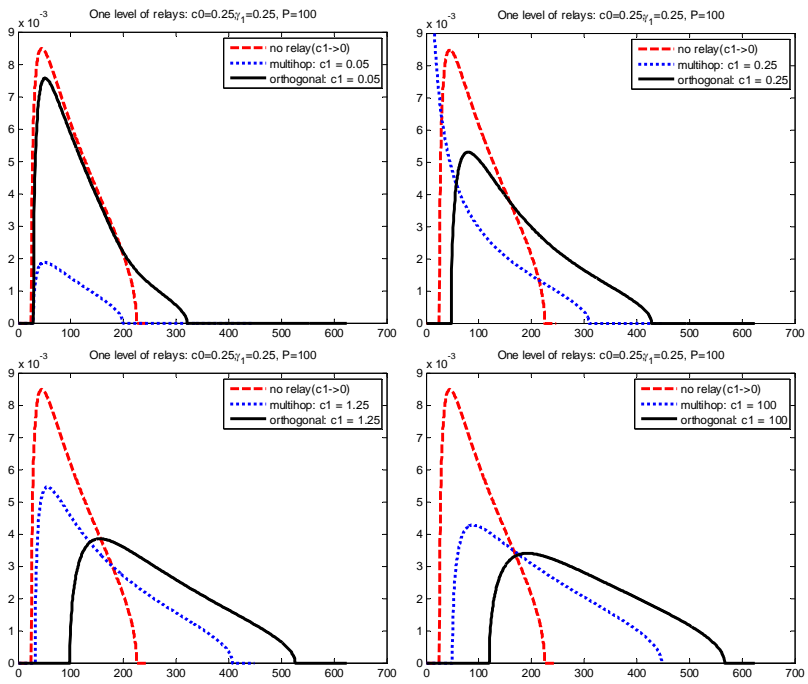

Fig. 6. Comparison between direct-link, multi-hop and orthogonal relay networks

\section{REFERENCES}

[1] V. A. Marčenko and L. A. Pastur, The Distribution of Eigenvalues in Certain Sets of Random Matrices, Math USSR-Sbornik, Vol. 72, No. 4 pp. 507-536, 1967.

[2] Z. D. Bai, Methodologies in Spectral Analysis of Large Dimensional Random Matrices. A Review, Statistica Sinica, Vol. 9, pp. 611-677, 1999.

[3] Z. D. Bai, J. .W. Silverstein, On the Empirical Distribution of Eigenvalues of a Class of Large Dimensional Random Matrices, Journal of Multivariate Analysis, Vol. 54, No. 2, pp. 175-192, 1995.

[4] J. W. Silverstein, Strong Convergence of the Empirical Distribution of Eigenvalues of Large Dimensional Random Matrices, Journal of Multivariate Analysis, Vol. 5, No. 11, pp. 331-339, 1995.

[5] S. Yeh and O. Leveque, Asymptotic Capacity of Multi-Level Amplifyand-Forward Relay Networks, 2007 IEEE International Symposium of Information Theory, Nice, June 2007.

[6] V. Morgenshtern and H. Bölcskei, Capacity of Large Amplify-andForward Relay Networks, 2006 IEEE Communication Theory Workshop, Puerto Rico, May 2006.

[7] V. Morgenshtern and H. Bölcskei, Crystallization in Large Wireless Networks, submitted to the IEEE Transactions on Information Theory.

[8] V. Morgenshtern and H. Bölcskei, Large Random Matrix Analysis of Relay Networks, Proceedings of the 2006 Allerton Conference.

[9] S. P. Borade, L. Zheng, and R. G. Gallager, Maximizing Degrees of Freedom in Wireless Networks, Allerton Annual Conference on Communication, Control and Computing, October 2003, pp. 561-570.

[10] I. E. Telatar, Capacity of Multi-Antenna Gaussian Channels, European Transactions on Telecommunications, vol. 10, no. 6, pp. 585-595, 1999.

[11] A. A. El Gamal and S. Zahedi, Capacity of a class of relay channels with orthogonal components, IEEE Trans. Inf. Theory, vol. 51, no. 5, pp. 1815-1817, May 2005. 